$11-1-2016$

\title{
Hierarchical Bayes Estimation of Reliability Indexes of Cold Standby Series System under General Progressive Type II Censoring Scheme
}

D. R. Barot

Department of Statistics, H. L. Institute of Commerce, Ahmedabad University, dinesh.barot@ahduni.edu.in

M. N. Patel

Department of Statistics, Gujarat University, Ahmedabad, mnpatel.stat@gmail.com

Follow this and additional works at: http://digitalcommons.wayne.edu/jmasm

Part of the Applied Statistics Commons, Social and Behavioral Sciences Commons, and the Statistical Theory Commons

\section{Recommended Citation}

Barot, D. R. and Patel, M. N. (2016) "Hierarchical Bayes Estimation of Reliability Indexes of Cold Standby Series System under General Progressive Type II Censoring Scheme," Journal of Modern Applied Statistical Methods: Vol. 15 : Iss. 2 , Article 39. DOI: $10.22237 /$ jmasm/1478003820

Available at: http://digitalcommons.wayne.edu/jmasm/vol15/iss2/39 


\section{Hierarchical Bayes Estimation of Reliability Indexes of Cold Standby Series System under General Progressive Type II Censoring Scheme}

\section{Cover Page Footnote}

We express our sincere thanks to the honorable Editors and anonymous Reviewers for valuable comments and fruitful suggestions, which significantly improved the manuscript. 


\section{Hierarchical Bayes Estimation of Reliability Indexes of Cold Standby Series System under General Progressive Type II Censoring Scheme}

\author{
D. R. Barot \\ Ahmedabad University \\ Ahmedabad, India
}

\author{
M. N. Patel \\ Gujarat University \\ Ahmedabad, India
}

In this paper, hierarchical Bayes approach is presented for estimation and prediction of reliability indexes and remaining lifetimes of a cold standby series system under general progressive Type II censoring scheme. A simulation study has been carried out for comparison purpose. The study will help reliability engineers in various industrial series system setups.

Keywords: Cold standby series system, general progressive Type II censoring, hierarchical Bayes estimation, Monte Carlo simulation

\section{Introduction}

A cold standby series system is widely applied to achieve high reliability in various engineering systems used in space exploration and satellite, textile manufacturing and carbon recovery systems. In such a series system, some units are placed in working mode while the rest in cold standby mode. When any unit in the working mode fails, it is replaced by any of the standby units in negligible time to survive the engineering system. The standby system becomes invalid when all standby units are used up, and one of the working units becomes unusable.

Mei, Liao, and Sun (1992) discussed the point estimation of reliability indexes by assuming that the life units in the series system have identical exponential distribution, and the failure rate is a known constant. Under the assumption that the failure rate is a random variable, $\mathrm{Su}$ and $\mathrm{Gu}$ (2003) derived the Bayes estimates while Bai, Yu, and $\mathrm{Hu}$ (1998) derived the multiple Bayes estimates of reliability indexes for the series system. Pham and Turkkan (1994) studied the reliability of

D. R. Barot is an Assistant Professor in the H. L. Institute of Commerce, Amrut Mody School of Management.Email him at:dinesh.barot@ahduni.edu.in. 


\section{BAROT \& PATEL}

the series system with Beta distribution component live. Willits (1997) studied reliability estimation of the series system using small binomial samples. Xu, Kang, and Shi (2002) discussed Bayesian and multiple Bayesian analysis of reliability performances for the series system. Barot and Patel (2014) derived the exact confidence limits of the reliability indexes for a cold standby series system under general progressive Type II censoring scheme using an empirical Bayesian approach.

In a life testing experiment, a censoring scheme that can balance between total times spent, number of units used and efficiency of statistical inference based on the results of an experiment is desirable. For this reason a more general censoring scheme called, general progressive Type II censoring scheme, has received a significant importance in the last few decades. This censoring scheme is extremely useful in both industrial life testing and clinical settings. The numerous articles dealing with inference procedures under this censoring scheme have been found in the journals (e.g., Balakrishnan \& Sandhu, 1996; Fernández, 2004; Kim \& Han, 2009; Barot \& Patel, 2014).

In Bayes approach, the posterior distribution of the parameters of interest given the data is obtained by assuming that the model hyper-parameter is known and then inferences are considered based on this distribution. However, when the information regarding the model hyper-parameter is unknown, empirical Bayes or hierarchical Bayes approaches are used to handle the super parameter structure for the estimation and prediction. In the empirical Bayes approach, the posterior distribution of the parameter of interest given the data is first obtained, assuming that the model hyper-parameters are known. The hyper-parameter is estimated from the marginal distribution of the data, and inferences are then based on the estimated posterior distribution.

However, in the case of non-availability of empirical data, estimates of parameters can be obtained through only an expert consulting. In such situations, hierarchical Bayes approach is more preferable than empirical Bayes approach. In hierarchical Bayes approach, a prior distribution of the hyper-parameter is specified according to expert's opinions, and then the posterior distribution of the parameter of interest is obtained. A parameter of interest is then estimated by its posterior mean and its precision is measured by its posterior variance. The hierarchical Bayes approach is straightforward and clear-cut, but computationally intensive, often involving high dimensional integration. It looks promising, but caution should be exercised in applying this approach. It has been described and applied extensively for various statistical inferences in literature (e.g., Han, 1998; Lehmann \& Casella, 


\section{HIERARCHICAL BAYES ESTIMATION OF RELIABILITY INDEXES}

1998; Papadopoulos, Tiwari, \& Zalkikar, 1996; Younes, Delampady, MacGibbon, \& Cherkaoui, 2007).

Statistical prediction was the most prevalent form of statistical inference, which is very important in a variety of disciplines such as medicine, engineering, and business. Various authors have studied the prediction problems in reliability and life testing problems (e.g., Dunsmore, 1974; Chhikara \& Guttman, 1982; Ali Mousa, 2001; Ali Mousa \& Jaheen, 2002).

Most of the research on a cold standby series system has focused on the usual Bayes approach. The objective of the present paper is to investigate estimation and prediction of reliability indexes and remaining lifetimes of the series system using a hierarchical Bayes approach under general progressive Type II censoring scheme.

\section{Bayes Estimation of Reliability Indexes}

In reliability and life testing studies, an exponential distribution is one of the most widely used lifetime models, and inference based on this distribution can be used quite effectively. A number of lifetime data have been analyzed, and it was observed that in most of the cases an exponential distribution provides a good fit. This distribution has been used to describe the life span of many items such as electronic tubes, light bulbs and mechanical components.

Suppose that a cold standby series system has $(k+n-1)$ identical units comprising a series of $k$ working units $U_{1}, U_{2}, \ldots, U_{k}$ being in an operational state and $(n-1)$ standby units $S_{1}, S_{2}, \ldots, S_{(n-1)}$ connected in a series. When any unit of the series of $k$ working units fails, any unit of $(n-1)$ standby units replaces it immediately through an alternation switch in negligible time, so that the series system stays operational. Figure 1 shows a functional diagram of the series system. Barot and Patel (2014) have considered such a series system and placed it on a life testing experiment under general progressive Type II censoring scheme by assuming that every unit has the failure rate $k \lambda$ with the probability density and cumulative distribution functions, respectively, as

$$
\mathrm{f}(x \mid \lambda)=\lambda k \mathrm{e}^{-\lambda k x}, \quad \lambda, x, k>0
$$

and

$$
\mathrm{F}(x \mid \lambda)=1-\mathrm{e}^{-\lambda k x}
$$




\section{BAROT \& PATEL}

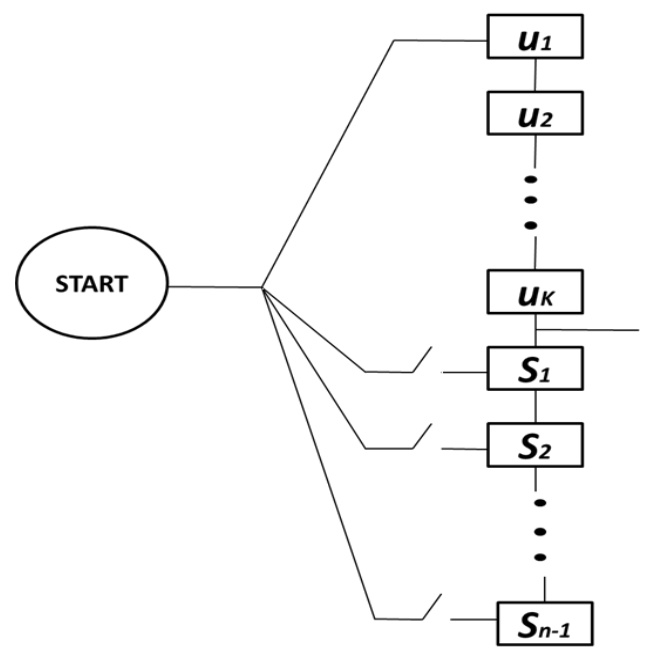

Figure 1. Cold standby series system with $(k+n-1)$ identical units

According to Cao and Cheng (1986), the reliability $\mathrm{R}(t)$ and average life $M T T F$ of the series system are strictly monotonic decreasing functions with respect to and can be given, respectively, by

$$
\mathrm{R}(t)=\sum_{i=0}^{n-1} \frac{\mathrm{e}^{-\lambda k t}(\lambda k t)^{i}}{i !}, \quad M T T F=\frac{n}{\lambda k}
$$

Under the general progressive Type II scheme, the lifetimes of the first $s$ units, i.e., $x_{(1)}, x_{(2)}, \ldots, x_{(s)}$ are not observed, and then the lifetimes until the $m^{\text {th }}$ failure, i.e., $x_{(s+1)}, x_{(s+2)}, \ldots, x_{(m)}$ are completely observed. At the time of every $i^{\text {th }}$ failure, $r_{i}$ units are randomly removed from the remaining $(n-s-1)$ standby units $(i=s+1$, $s+2, \ldots, m-1)$. Instead of continuing the test until the entire standby units are used up, the test is terminated at the time of the $m^{\text {th }}$ failure $(m<n)$, and all the remaining $r_{m}$ standby units are removed from the test, where $r_{m}$ is given by

$$
r_{m}=n-m-1-\sum_{i=s+1}^{m-1} r_{i}
$$

Following Barot and Patel (2014), the likelihood function based on the general progressive Type II sample $\mathbf{x}=\left(x_{(s+1)}, x_{(s+2)}, \ldots, x_{(m)}\right)$ can be written as 


\section{HIERARCHICAL BAYES ESTIMATION OF RELIABILITY INDEXES}

$$
\mathrm{L}(\mathbf{x} \mid \lambda)=A \lambda^{m-s} k^{m-s}\left(1-\mathrm{e}^{-\lambda k x_{(s+1)}}\right)^{s} \mathrm{e}^{-\lambda k w}
$$

where

$$
A=\left(\begin{array}{c}
n-1 \\
s
\end{array}\right)(n-s-2) \prod_{j=s+2}^{m}\left(n-j-1-\sum_{i=s+1}^{j-1} r_{i}\right), \quad w=\sum_{i=s+1}^{m}\left(1+r_{i}\right) x_{(i)}
$$

The concern in Bayesian estimation is an appropriate choice of a prior distribution for a parameter to consider subjective information from experienced experts. An exponential distribution is one of most prominent random probability distributions, and its good mathematical properties facilitate insight and computational reduction. In reliability analysis and life testing, it is preferred over many other distributions due to its richness, computational ease, better fit to the failure data, analytical tractability, and easy interpretability. To ease the computational burden and get computable closed form expression for the posterior distribution, it is assumed that the unknown failure rate $\lambda$ is the realization of a random variable and follows an exponential prior with the probability density function

$$
\pi(\lambda \mid \beta)=\beta \mathrm{e}^{-\beta \lambda}, \quad \lambda>0
$$

The likelihood function (4) and prior distribution (5) can be easily combined to form a posterior distribution that represents total knowledge about the parameter $\lambda$ after the data have been observed. It is

$$
\pi(\lambda \mid \mathbf{x})=\frac{\lambda^{m-s}\left[1-\mathrm{e}^{-\lambda k x_{(s+1)}}\right]^{2} \mathrm{e}^{-\lambda(k w+\beta)}}{\Gamma(m-s+1) D_{s}}
$$

where

$$
D_{s}=\sum_{j=0}^{s}\left(\begin{array}{l}
s \\
j
\end{array}\right)(-1)^{j} \frac{1}{\left[k w_{j}+\beta\right]^{m-s+1}} ; \quad w_{j}=w+x_{(s+1)} j
$$




\section{BAROT \& PATEL}

In Bayesian analysis, a loss function must be specified in order to obtain Bayes estimates. The loss function is a non-negative function of the distance between the estimate and the true value. When decisions become gradually more

damaging for large errors, the use of squared error loss function, $\mathrm{L}(\hat{\lambda}, \lambda)=(\hat{\lambda}-\lambda)^{2}$, is more appropriate because of its analytical tractability. The Bayes estimate of parameter $\lambda$, reliability $\mathrm{R}(t)$ and $M T T F$ can be obtained under the squared error loss function, respectively, as

$$
\begin{gathered}
\hat{\lambda}_{B}=\frac{(m-s+1) D_{S 1}}{D_{S}} \\
\hat{\mathrm{R}}(t)_{B}=\frac{1}{\Gamma(m-s+1) D_{S}} \sum_{i=0}^{n-1} \frac{(k t)^{i} \Gamma(m-2+i+1)}{i !} D_{S 2(i)} \\
\operatorname{MTTF}_{B}=\frac{n D_{S 3}}{k(m-s) D_{S}}
\end{gathered}
$$

where

$$
\begin{gathered}
D_{S 1}=\sum_{j=0}^{s}\left(\begin{array}{l}
s \\
j
\end{array}\right)(-1)^{j} \frac{1}{\left[k w_{j}+\beta\right]^{m-s+2}} ; \quad D_{S 2(i)}=\sum_{j=0}^{s}\left(\begin{array}{c}
s \\
j
\end{array}\right)(-1)^{j} \frac{1}{\left(k t+k w_{j}+\beta\right)^{m-s+i+1}} \\
D_{S 3}=\sum_{j=0}^{s}\left(\begin{array}{c}
s \\
j
\end{array}\right)(-1)^{j} \frac{1}{\left(k w_{j}+\beta\right)^{m-s}}
\end{gathered}
$$

\section{Hierarchical Bayesian Analysis}

The idea in a Bayesian model is that when you look at a likelihood function and decide right priors for parameters. Instead, it may be more appropriate to use priors depending on other parameters those are not mentioned in a likelihood function. These parameters themselves will require priors and can depend on new ones. This can continue in a hierarchical framework until there are no more parameters to incorporate in the model. In this section, hierarchical Bayes estimates of reliability indexes of the series system are constructed. 


\section{HIERARCHICAL BAYES ESTIMATION OF RELIABILITY INDEXES}

Due to the complicity of practical problems and uncertainty about the true level of an expert, it is quite difficult to give the exact estimate of a super parameter $\beta$. However, the value of $\beta$ can be obtained in an approximate interval denoted by $(a, b)$ through an expert consulting. As there is no other information on the parameter $\beta$, it is assumed that it has uniform distribution on $(a, b)$ with the probability density function

$$
\mathrm{U}(\beta \mid a, b)=\frac{1}{b-a}
$$

given in $\mathrm{Xu}$ et al. (2002). In order to obtain the posterior density of $\beta$ given $\mathbf{x}$,

$$
\begin{aligned}
\int_{a}^{b} \mathrm{U}(\beta \mid a, b) \mathrm{m}(\mathbf{x} \mid \beta) d \beta & =\frac{\Gamma(m-s+1)}{b-a} A k^{m-s} \sum_{j=0}^{s}\left(\begin{array}{c}
s \\
j
\end{array}\right)(-1)^{j} \int_{a}^{b} \frac{\beta}{\left(k w_{j}+\beta\right)^{m-s+1}} d \beta \\
& =\frac{A k^{m-s} \Gamma(m-s-1)}{a-b} D_{S 4}
\end{aligned}
$$

where

$$
D_{S 4}=\sum_{j=0}^{s}\left(\begin{array}{l}
s \\
j
\end{array}\right)(-1)^{j}\left[\left(k w_{j}+b\right)^{-m+s}\left(k w_{j}+b m-b s\right)-\left(k w_{j}+a\right)^{-m+s}\left(k w_{j}+a m-a s\right)\right]
$$

From Bayes theorem, the posterior density of $\beta$ given $\mathbf{x}$ can be obtained as

$$
\mathrm{h}(\beta \mid \mathbf{x})=\frac{\mathrm{U}(\beta \mid a, b) \mathrm{m}(\mathbf{x} \mid \beta)}{\int_{a}^{b} \mathrm{U}(\beta \mid a, b) \mathrm{m}(\mathbf{x} \mid \beta) d \beta}=\frac{-\beta(m-s)(m-s-1) D_{S}}{D_{S 4}}
$$

Under the squared error loss function, the Bayes estimate of $\beta$ can be given by

$$
\hat{\beta}_{B}=\mathrm{E}^{h}(\beta \mid \mathbf{x})=\int_{a}^{b} \beta \mathrm{h}(\beta \mid \mathbf{x}) d \beta=\frac{D_{S 5}-D_{S 6}}{(m-s-2) D_{S 4}}
$$

where 


\section{BAROT \& PATEL}

$$
\begin{aligned}
& D_{S 5}=\sum_{j=0}^{s}\left(\begin{array}{l}
s \\
j
\end{array}\right)(-1)^{j}\left\{\frac{2 k^{2} w_{j}^{2}+2 b k(m-s) w_{j}+(m-s)(m-s-1) b^{2}}{\left(k w_{j}+b\right)^{m-s}}\right\} \\
& D_{S 6}=\sum_{j=0}^{s}\left(\begin{array}{l}
s \\
j
\end{array}\right)(-1)^{j}\left\{\frac{2 k^{2} w_{j}^{2}+2 a k(m-s) w_{j}+(m-s)(m-s-1) a^{2}}{\left(k w_{j}+a\right)^{m-s}}\right\}
\end{aligned}
$$

Using (10) in (7), (8), and (9), the hierarchical Bayes estimates of $\lambda, \mathrm{R}(t)$ and MTTF under the squared error loss function can be obtained as follows:

$$
\begin{gathered}
\hat{\lambda}_{H B}=\frac{(m-s+1) D_{S 7}}{D_{S^{\prime}}} \\
\hat{\mathrm{R}}(t)_{H B}=\frac{1}{\Gamma(m-s+1) D_{S^{\prime}}} \sum_{i=0}^{n-1} \frac{(k t)^{i} \Gamma(m-s+i+1)}{i !} D_{S 8(i)} \\
\operatorname{MTTF}_{H B}=\frac{n D_{S 9}}{k(m-s) D_{S^{\prime}}}
\end{gathered}
$$

where

$$
\begin{gathered}
D_{S^{\prime}}=\sum_{j=0}^{s}\left(\begin{array}{l}
s \\
j
\end{array}\right)(-1)^{j} \frac{1}{\left[k w_{j}+\hat{\beta}_{B}\right]^{m-s+1}} ; \quad D_{S 7}=\sum_{j=0}^{s}\left(\begin{array}{l}
s \\
j
\end{array}\right)(-1)^{j} \frac{1}{\left[k w_{j}+\hat{\beta}_{B}\right]^{m-s+2}} ; \\
D_{S 8(i)}=\sum_{j=0}^{s}\left(\begin{array}{c}
s \\
j
\end{array}\right)(-1)^{j} \frac{1}{\left(k t+k w_{j}+\hat{\beta}_{B}\right)^{m-s+i+1}} ; \quad D_{S 9}=\sum_{j=0}^{s}\left(\begin{array}{c}
s \\
j
\end{array}\right)(-1)^{j} \frac{1}{\left[k w_{j}+\hat{\beta}_{B}\right]^{m-s}} ;
\end{gathered}
$$

In Bayesian inference, the 100(1- $\alpha) \%$ highest probability density (HPD) interval of the parameter of interest is the shortest interval in parameter space that contains $100(1-\alpha) \%$ of the probable values of the parameter. It is one of the most useful tools to measure posterior uncertainty that includes more probable values and excludes the least probable values of the parameter. Since the posterior 


\section{HIERARCHICAL BAYES ESTIMATION OF RELIABILITY INDEXES}

distribution (6) is unimodal, the $100(1-\alpha) \%$ Bayes HPD-interval $\left(p_{1}, p_{2}\right)$ for $\lambda$ must simultaneously satisfy the equations

$$
\begin{gathered}
\pi^{*}\left(p_{1} \mid \mathbf{x}\right)=\pi^{*}\left(p_{2} \mid \mathbf{x}\right) \\
\int_{p_{1}}^{p_{2}} \pi^{*}(\lambda \mid \mathbf{x}) d \lambda=1-\alpha
\end{gathered}
$$

After tedious algebra, the equations (14) and (15) can be written in the form

$$
\left(\frac{p_{1}}{p_{2}}\right)^{m-s} \mathrm{e}^{\left(p_{2}-p_{1}\right)(k w+\beta)}=\left(\frac{1-\mathrm{e}^{-p_{2} k x_{(s+1)}}}{1-\mathrm{e}^{-p_{1} k x_{(s+1)}}}\right)
$$

and

$$
\frac{1}{D_{S}} \sum_{j=0}^{s}\left(\begin{array}{l}
s \\
j
\end{array}\right)(-1)^{j} c_{j}^{-m+s-1}\left[\mathrm{e}^{-c_{j} p_{1}} \sum_{j_{1}=0}^{m-s} \frac{\left(c_{j} p_{1}\right)^{j_{1}}}{j_{1} !}-\mathrm{e}^{-c_{j} p_{2}} \sum_{j_{1}=0}^{m-s} \frac{\left(c_{j} p_{2}\right)^{j_{1}}}{j_{1} !}\right]=1-\alpha
$$

where $c_{j}=k w_{j}+\beta$.

The 100(1- $\alpha) \%$ Bayes HPD-intervals of $\mathrm{R}(t)$ and MTTF can be obtained from (8) and (9). When the super parameter $\beta$ is unknown, the $100(1-\alpha) \%$ hierarchical Bayes HPD-intervals of reliability indexes can be obtained by using the estimate $\hat{\beta}_{B}$ for $\beta$.

\section{Prediction of Remaining Lifetimes Truncated at $X_{(m)}$}

The prediction of remaining lifetimes, based on a current available sample, known as an informative sample, is an important feature in Bayesian analysis. Howlader (1985) presented HPD-prediction intervals for the $z^{\text {th }}$ order statistic of a future sample. Fernández (2000) considered the problem of predicting an independent future sample from the Rayleigh distribution under doubly Type II censoring scheme. Raqab and Madi (2002) considered an estimation of the predictive distribution of the total time on a test up to certain failures in a future sample, as well as that of the remaining testing time until all the units in the original sample have failed. 


\section{BAROT \& PATEL}

Let

$$
x_{(l)}, \quad m+1 \leq l \leq n_{1}, n_{1}=n-\sum_{i=s+1}^{m-1} r_{i}
$$

denote the lifetime of the $l^{\text {th }}$ unit to fail. The conditional probability density function of $y=x_{(l)}-x_{(m)}$ from the probability density function truncated at $x_{(m)}$ is given by

$$
\mathrm{f}_{1}(y \mid \lambda)=\frac{[\mathrm{F}(y \mid \lambda)]^{l-m-1}[1-\mathrm{F}(y \mid \lambda)]^{n_{1}-l} \mathrm{f}(y \mid \lambda)}{\mathrm{B}\left(l-m, n_{1}-l+1\right)}, \quad y \geq 0
$$

From (1) and (2), the function $\mathrm{f}_{1}=(y \mid \lambda)$ can be obtained as

$$
\mathrm{f}_{1}(y \mid \lambda)=\frac{\lambda k \mathrm{e}^{-\lambda k y\left(n_{1}-l+1\right)}\left(1-\mathrm{e}^{-\lambda k y}\right)^{l-m-1}}{\mathrm{~B}\left(l-m, n_{1}-l+1\right)}
$$

Based on the general progressive Type II censored sample $\mathbf{x}$, the conditional joint probability density function of $y$ and $\lambda$ can be written as

$$
\begin{aligned}
\mathrm{g}_{1}(y, \lambda \mid \mathbf{x}) & =\mathrm{f}_{1}(y \mid \lambda) \pi^{*}(\lambda \mid \mathbf{x}) \\
& =\frac{k \lambda^{m-s+1} \mathrm{e}^{-\lambda\left[k y\left(n_{1}-l+1\right)+k w+\beta\right]}\left(1-\mathrm{e}^{-\lambda k y}\right)^{l-m+1}\left(1-\mathrm{e}^{-\lambda k x_{(s+1)}}\right)^{s}}{\Gamma(m-s+1) \mathrm{B}\left(l-m, n_{1}-l+1\right) D_{S}}
\end{aligned}
$$

The Bayes predictive density function of $y$ can be obtained as

$$
\begin{aligned}
\mathrm{p}(y \mid \mathbf{x}) & =\int_{0}^{\infty} \mathrm{g}_{1}(y, \lambda \mid \mathbf{x}) d \lambda \\
& =k_{1} \Gamma(m-s+2) \sum_{p_{1}=0}^{l-m-1} \sum_{q_{1}=0}^{s} \frac{\left(\begin{array}{c}
l-m-1 \\
p_{1}
\end{array}\right)\left(\begin{array}{l}
s \\
q_{1}
\end{array}\right)(-1)^{p_{1}+q_{1}}}{w_{p_{1} q_{1}}^{m-s+2}}
\end{aligned}
$$

where 


\section{HIERARCHICAL BAYES ESTIMATION OF RELIABILITY INDEXES}

$$
\begin{aligned}
k_{1} & =\frac{k}{\Gamma(m-s+1) \mathrm{B}\left(l-m, n_{1}-l+1\right) D_{s}} \\
w_{p_{1} q_{1}} & =k\left(n_{1}-l+1+p_{1}\right) y+k w-k x_{(s+1)} q_{1}+\beta
\end{aligned}
$$

Under the squared error loss function, the Bayes predictive estimate of $y$ can be obtained as

$$
y^{*}=\frac{\sum_{p_{1}=0}^{l-m-1} \sum_{q_{1}=0}^{s}\left(\begin{array}{c}
l-m-1 \\
p_{1}
\end{array}\right)\left(\begin{array}{c}
s \\
q_{1}
\end{array}\right)(-1)^{p_{1}+q_{1}} \frac{\left(k w+k x_{(s+1)} q_{1}+\beta\right)^{-m+s}}{\left(n_{1}-l+1+p_{1}\right)^{2}}}{k(m-s) \mathrm{B}\left(l-m, n_{1}-l+1\right) D_{s}}
$$

Thus, the Bayes predictive estimate of $x(l)$ can be given by

$$
x_{(l)}^{*}=x_{(m)}+y^{*}
$$

Moreover, the 100(1- $\alpha) \%$ Bayes HPD-prediction interval of $y^{*}$ is given by $\left(h_{1}, h_{2}\right)$, where $h_{1}$ and $h_{2}$ are solutions of the equations

$$
\mathrm{p}\left(h_{1} \mid \mathbf{x}\right)=\mathrm{p}\left(h_{2} \mid \mathbf{x}\right)
$$

and

$$
\mathrm{p}\left(h_{1}<y<h_{2}\right)=1-\alpha
$$

Using (19) in (20) and (21), after tedious algebra, we have

$$
\sum_{p_{1}=0}^{l-m-1} \sum_{q_{1}=0}^{s} \frac{\left(\begin{array}{c}
l-m-1 \\
p_{1}
\end{array}\right)\left(\begin{array}{c}
s \\
q_{1}
\end{array}\right)(-1)^{p_{1}+q_{1}}}{\delta_{p_{1} q_{1}}^{m-s+2}}=\sum_{p_{1}=0}^{l-m-1} \sum_{q_{1}=0}^{s} \frac{\left(\begin{array}{c}
l-m-1 \\
p_{1}
\end{array}\right)\left(\begin{array}{c}
s \\
q_{1}
\end{array}\right)(-1)^{p_{1}+q_{1}}}{\gamma_{p_{1} q_{1}}^{m-s+2}}
$$

and 


$$
\frac{-\sum_{p_{1}=0}^{l-m-1} \sum_{q_{1}=0}^{s}\left(\begin{array}{c}
l-m-1 \\
p_{1}
\end{array}\right)\left(\begin{array}{c}
s \\
q_{1}
\end{array}\right)(-1)^{p_{1}+q_{1}} \frac{\left(\gamma_{p_{1} q_{1}}^{-m+s-1}-\delta_{p_{1} q_{1}}^{-m+s-1}\right)}{n_{1}-l+p_{1}+1}}{\mathrm{~B}\left(l-m, n_{1}-l+1\right) D_{S}}=1-\alpha
$$

where

$$
\begin{aligned}
& \delta_{p_{1} q_{1}}=k\left(n_{1}-l+p_{1}+1\right) h_{1}+k w+k x_{(s+1)} q_{1}+\beta \\
& \gamma_{p_{1} q_{1}}=k\left(n_{1}-l+p_{1}+1\right) h_{2}+k w+k x_{(s+1)} q_{1}+\beta
\end{aligned}
$$

Hence, the $100(1-\alpha) \%$ Bayes HPD-prediction interval for $x_{(l)}$ is

$$
\left(x_{(m)}+h_{1}, x_{(m)}+h_{2}\right)
$$

When the super parameter $\beta$ is unknown, the hierarchical Bayes predictive estimates $x_{(l)}$ and the corresponding 100(1- 1$) \%$ hierarchical HPD-prediction interval of can be obtained by using the estimate $\hat{\beta}_{B}$ for $\beta$ in (19) and (24).

\section{Simulation Study}

An extensive Monte Carlo simulation study was carried out to illustrate and compare the performance of hierarchical Bayes estimates of reliability indexes of the system with series of $k$ units in working mode and $(n-1)$ units in cold standby mode. The performance is evaluated based on estimated risks and biases for different combinations of sample size $(n)$, effective sample size $(m-s)$, and general progressive Type II censoring scheme $\mathbf{r}=\left(r_{s+1}, r_{s+2}, \ldots, r_{m}\right)$. The different censoring schemes applied in the simulation study are summarized in Table 1.

For given values $a=0, b=1$ and 100,00,000 generated uniform numbers, two values of $\beta$, one is the true value $\beta_{T}=0.5002$ and another is the expert value $\beta_{E}=0.4999$ were obtained by the Monte Carlo means. The corresponding $\lambda=2.0008$ is brought from the prior (5) and the expert value $\beta_{E}$. Using the generated value of $\lambda$, we have generated a general progressive Type II censored sample $\mathbf{x}=\left(x_{(s+1)}, x_{(s+2)}, \ldots, x_{(m)}\right)$ with the censoring scheme $\mathbf{r}$ from the exponential distribution according to the algorithm presented in Balakrishnan and Sandhu (1996) that involves the following steps: 


\section{HIERARCHICAL BAYES ESTIMATION OF RELIABILITY INDEXES}

1. Generate $V_{m}$ from the Beta distribution with parameters $(n-s)$ and $(s+1)$

2. Independently generate $Z_{s+i}$ from $\mathrm{U}(0,1)$ and then set $V_{s+i}=Z_{s+i}^{\frac{1}{a_{s+i}}}$, $a_{s+i}=i+\sum_{j=m-i+1}^{m} r_{j}$ for $\mathrm{i}=1,2, \ldots,(m-s-1)$

3. Set $U_{s+1}=1-V_{m}$ and $U_{s+i}=1-\left(V_{m-i+1} V_{m-i+2} \ldots V_{m}\right)$, $i=2,3, \ldots,(m-s)$

4. For the generated value of $\lambda$ and given $k, x_{(s+i)}=-\frac{1}{\lambda k} \ln \left(1-U_{s+i}\right)$, $i=1,2, \ldots,(m-s)$ is the required general progressive Type II censored sample of size $(m-s)$ from the exponential distribution

The Bayes estimates, hierarchical Bayes estimates, and the corresponding estimated risks were computed by averaging over 100,000 simulations, and are reported, respectively, in Tables 2-6. From the simulation results, the following points can be drawn:

1) For the fixed sample size $n$ and initial $s$ unobserved failures, as the predetermined number of failures $m$ increases, the estimated risks of estimates of reliability indexes decrease, that is, the performance becomes better in terms of the estimated risks. (Refer to Tables 2-4)

2) For the fixed effective sample size $(m-s)$, the estimated risks of estimates of failure rate $\lambda$ and reliability $\mathrm{R}(t)$ decrease while that of $M T T F$ increase with the increasing sample size $n$. (Refer to Tables 24)

3) For the fixed sample size $n$ and predetermined number of failures $m$, the estimated risks of estimates of failure rate $\lambda$ and reliability $\mathrm{R}(t)$ increase while that of MTTF decrease with the increasing number of initial $s$ unobserved failures. (Refer to Tables 2-4)

4) For the fixed sample size $n$ and effective sample size $(m-s)$, the estimated risks of the estimates of MTTF decrease while that of reliability $\mathrm{R}(t)$ decrease for small sample size and increase for moderate and large sample sizes with increasing number of working units $k$. (Refer to Table 6)

5) It is noted that an increase in $k$ does not have any dampening effect on the estimated risk of failure rate $\lambda$. (Refer to Table 6) 


\section{BAROT \& PATEL}

6) The estimated risks of the Bayes estimates of reliability indexes are smaller than the corresponding hierarchical Bayes estimates for all the considered cases. This indicates that Bayes estimates outperform the hierarchical Bayes estimates. (Refer to Tables 2-4)

7) For the fixed effective sample size $(m-s)$, as the sample size $n$ increases, the Bayes and hierarchical Bayes estimates of failure rate $\lambda$ decrease while reliability $\mathrm{R}(t)$ and $M T T F$ increase, i.e., the series system survives for a long period. (Refer to Tables 2-4)

8) For the fixed sample size $n$ and effective sample size $(m-s)$, as the number of working units $k$ increases, the Bayes and hierarchical Bayes estimates of reliability $\mathrm{R}(t)$ and $M T T F$ decrease, i.e., the series system fails frequently. (Refer to Table 5)

Table 1. Progressive Type II censoring schemes (CS) applied to the simulation study

\begin{tabular}{|c|c|c|c|c|c|c|c|c|c|}
\hline$n$ & $m$ & $s$ & CS No. & $r=\left(r_{s+1}, r_{s+2}, \ldots, r_{m}\right)$ & $n$ & $m$ & $s$ & CS No. & $r=\left(r_{s+1}, r_{s+2}, \ldots, r_{m}\right)$ \\
\hline \multirow[t]{12}{*}{20} & 8 & 3 & [1] & $(1,0,4,1,6)$ & 50 & 10 & 3 & [19] & $(6,8,10,4,3,7,2)$ \\
\hline & & & [2] & $(0,0,0,0,12)$ & & & & [20] & $(0,0,0,0,0,0,40)$ \\
\hline & & & [3] & $(12,0,0,0,0)$ & & & & [21] & $(40,0,0,0,0,0,0)$ \\
\hline & & 4 & [4] & $(2,0,4,6)$ & & & 4 & [22] & $(6,8,10,4,5,7)$ \\
\hline & & & [5] & $(0,0,0,12)$ & & & & [23] & $(0,0,0,0,0,40)$ \\
\hline & & & [6] & $(12,0,0,0)$ & & & & [24] & $(40,0,0,0,0,0)$ \\
\hline & 10 & 3 & [7] & $(2,0,3,0,1,2,2)$ & 100 & 8 & 3 & [25] & $(16,12,20,14,30)$ \\
\hline & & & [8] & $(0,0,0,0,0,0,10)$ & & & & [26] & $(0,0,0,0,92)$ \\
\hline & & & [9] & $(10,0,0,0,0,0,0)$ & & & & [27] & $(92,0,0,0,0)$ \\
\hline & & 4 & [10] & $(3,0,2,1,0,4)$ & & & 4 & [28] & $(28,25,17,22)$ \\
\hline & & & {$[11]$} & $(0,0,0,0,0,10)$ & & & & [29] & $(0,0,0,92)$ \\
\hline & & & [12] & $(10,0,0,0,0,0)$ & & & & [30] & $(92,0,0,0)$ \\
\hline \multirow[t]{6}{*}{50} & 8 & 3 & [13] & $(6,12,11,4,9)$ & & 10 & 3 & [31] & $(6,13,15,14,8,12,22)$ \\
\hline & & & [14] & $(0,0,0,0,42)$ & & & & [32] & $(0,0,0,0,0,0,90)$ \\
\hline & & & [15] & $(42,0,0,0,0)$ & & & & [33] & $(90,0,0,0,0,0,0)$ \\
\hline & & 4 & {$[16]$} & $(8,15,7,12)$ & & & 4 & [34] & $(16,18,15,14,15,12)$ \\
\hline & & & {$[17]$} & $(0,0,0,42)$ & & & & [35] & $(0,0,0,0,0,90)$ \\
\hline & & & [18] & $(42,0,0,0)$ & & & & [36] & $90,0,0,0,0,0$ \\
\hline
\end{tabular}




\section{HIERARCHICAL BAYES ESTIMATION OF RELIABILITY INDEXES}

Table 2. Estimates of failure rate of $\lambda$ and their estimated risks

\begin{tabular}{|c|c|c|c|c|c|}
\hline CS & $\hat{\boldsymbol{\beta}}$ & $\hat{\lambda}_{B}$ & $\hat{\lambda}_{H B}$ & $\operatorname{ER}\left(\hat{\lambda}_{B}\right)$ & $\operatorname{ER}\left(\hat{\lambda}_{H B}\right)$ \\
\hline 1 & 0.5257 & 2.2951 & 2.3031 & 0.6755 & 0.7867 \\
\hline 2 & 0.5273 & 2.2706 & 2.2772 & 0.6508 & 0.7554 \\
\hline 3 & 0.5078 & 2.5724 & 2.6029 & 1.0682 & 1.3812 \\
\hline 4 & 0.5229 & 2.3396 & 2.3504 & 0.7288 & 0.8545 \\
\hline 5 & 0.5250 & 2.3066 & 2.3154 & 0.6917 & 0.8070 \\
\hline 6 & 0.5005 & 2.6863 & 2.7278 & 1.2986 & 1.6107 \\
\hline 7 & 0.5234 & 2.3322 & 2.3365 & 0.6178 & 0.6879 \\
\hline 8 & 0.5280 & 2.2616 & 2.2629 & 0.5479 & 0.6060 \\
\hline 9 & 0.5098 & 2.5440 & 2.5598 & 0.8976 & 1.0207 \\
\hline 10 & 0.5192 & 2.3984 & 2.4059 & 0.6934 & 0.7772 \\
\hline 11 & 0.5246 & 2.3146 & 2.3181 & 0.5986 & 0.6655 \\
\hline 12 & 0.4985 & 2.7215 & 2.7505 & 1.2238 & 1.4206 \\
\hline 13 & 0.5279 & 2.2612 & 2.2676 & 0.6451 & 0.7537 \\
\hline 14 & 0.5300 & 2.2301 & 2.2348 & 0.6168 & 0.7176 \\
\hline 15 & 0.5078 & 2.5717 & 2.6025 & 1.0667 & 1.2980 \\
\hline 16 & 0.5269 & 2.2779 & 2.2850 & 0.6593 & 0.7669 \\
\hline 17 & 0.5292 & 2.2422 & 2.2473 & 0.6250 & 0.7233 \\
\hline 18 & 0.5006 & 2.6853 & 2.7265 & 1.2945 & 1.6056 \\
\hline 19 & 0.5269 & 2.2790 & 2.2808 & 0.5607 & 0.6197 \\
\hline 20 & 0.5317 & 2.2033 & 2.2042 & 0.4952 & 0.5432 \\
\hline 21 & 0.5098 & 2.5438 & 2.5593 & 0.8921 & 1.0120 \\
\hline 22 & 0.5265 & 2.2845 & 2.2865 & 0.5639 & 0.6223 \\
\hline 23 & 0.5307 & 2.2195 & 2.2199 & 0.5060 & 0.5548 \\
\hline 24 & 0.4985 & 2.7209 & 2.7495 & 1.2191 & 1.4100 \\
\hline 25 & 0.5299 & 2.2310 & 2.2358 & 0.6177 & 0.7183 \\
\hline 26 & 0.5305 & 2.2219 & 2.2262 & 0.6038 & 0.6983 \\
\hline 27 & 0.5076 & 2.5706 & 2.6017 & 1.0657 & 1.2978 \\
\hline 28 & 0.5286 & 2.2511 & 2.2564 & 0.6259 & 0.7249 \\
\hline 29 & 0.5301 & 2.2282 & 2.2323 & 0.6054 & 0.6989 \\
\hline 30 & 0.5004 & 2.6824 & 2.7254 & 1.2902 & 1.5969 \\
\hline 31 & 0.5317 & 2.2031 & 2.2040 & 0.4944 & 0.5427 \\
\hline 32 & 0.5326 & 2.1878 & 2.1883 & 0.4845 & 0.5312 \\
\hline 33 & 0.5098 & 2.5424 & 2.5588 & 0.8907 & 1.0105 \\
\hline 34 & 0.5296 & 2.2362 & 2.2365 & 0.5266 & 0.5806 \\
\hline 35 & 0.5321 & 2.1971 & 2.1970 & 0.4951 & 0.5437 \\
\hline 36 & 0.4985 & 2.7203 & 2.7403 & 1.2161 & 1.4044 \\
\hline
\end{tabular}




\section{BAROT \& PATEL}

Table 3. Estimates of reliability $R(t)$ and their estimated risks

\begin{tabular}{|c|c|c|c|c|c|}
\hline CS & $R(t)$ & $\hat{\mathbf{R}}(\boldsymbol{t})_{B}$ & $\hat{\mathbf{R}}(t)_{H B}$ & $\operatorname{ER}\left(\hat{\mathbf{R}}(t)_{B}\right)$ & $\operatorname{ER}\left(\hat{\mathbf{R}}(t)_{H B}\right)$ \\
\hline 1 & 0.4695 & 0.4554 & 0.4600 & 0.0598 & 0.0623 \\
\hline 2 & & 0.4638 & 0.4686 & 0.0601 & 0.0617 \\
\hline 3 & & 0.3692 & 0.3717 & 0.0646 & 0.0661 \\
\hline 4 & & 0.4410 & 0.4453 & 0.0600 & 0.0625 \\
\hline 5 & & 0.4520 & 0.4564 & 0.0603 & 0.0626 \\
\hline 6 & & 0.3410 & 0.3428 & 0.0789 & 0.0815 \\
\hline 7 & & 0.4249 & 0.4289 & 0.0586 & 0.0604 \\
\hline 8 & & 0.4505 & 0.4549 & 0.0583 & 0.0601 \\
\hline 9 & & 0.3552 & 0.3578 & 0.0642 & 0.0659 \\
\hline 10 & & 0.4019 & 0.4055 & 0.0596 & 0.0613 \\
\hline 11 & & 0.4311 & 0.4352 & 0.0584 & 0.0602 \\
\hline 12 & & 0.3060 & 0.3075 & 0.0734 & 0.0752 \\
\hline 13 & 0.9999 & 0.9643 & 0.9676 & 0.0069 & 0.0092 \\
\hline 14 & & 0.9665 & 0.9696 & 0.0063 & 0.0085 \\
\hline 15 & & 0.9361 & 0.9429 & 0.0144 & 0.0195 \\
\hline 16 & & 0.9631 & 0.9666 & 0.0071 & 0.0095 \\
\hline 17 & & 0.9657 & 0.9689 & 0.0065 & 0.0087 \\
\hline 18 & & 0.9219 & 0.9307 & 0.0190 & 0.0259 \\
\hline 19 & & 0.9722 & 0.9745 & 0.0048 & 0.0063 \\
\hline 20 & & 0.9768 & 0.9786 & 0.0039 & 0.0050 \\
\hline 21 & & 0.9513 & 0.9558 & 0.0101 & 0.0131 \\
\hline 22 & & 0.9719 & 0.9742 & 0.0049 & 0.0064 \\
\hline 23 & & 0.9759 & 0.9778 & 0.0040 & 0.0052 \\
\hline 24 & & 0.9318 & 0.9387 & 0.0159 & 0.0209 \\
\hline 25 & 1.0000 & 0.9993 & 0.9997 & $1.136 \times 10^{-5}$ & $1.105 \times 10^{-5}$ \\
\hline 26 & & 0.9993 & 0.9997 & $1.079 \times 10^{-5}$ & $1.172 \times 10^{-5}$ \\
\hline 27 & & 0.9981 & 0.9991 & $6.165 \times 10^{-5}$ & $3.532 \times 10^{-5}$ \\
\hline 28 & & 0.9993 & 0.9996 & $1.934 \times 10^{-5}$ & $1.141 \times 10^{-5}$ \\
\hline 29 & & 0.9993 & 0.9997 & $1.798 \times 10^{-5}$ & $1.262 \times 10^{-5}$ \\
\hline 30 & & 0.9973 & 0.9988 & $8.788 \times 10^{-5}$ & $5.091 \times 10^{-5}$ \\
\hline 31 & & 0.9997 & 0.9999 & $6.051 \times 10^{-5}$ & $3.037 \times 10^{-5}$ \\
\hline 32 & & 0.9997 & 0.9999 & $5.034 \times 10^{-5}$ & $2.081 \times 10^{-5}$ \\
\hline 33 & & 0.9993 & 0.9996 & $2.221 \times 10^{-5}$ & $1.098 \times 10^{-5}$ \\
\hline 34 & & 0.9997 & 0.9998 & $6.072 \times 10^{-5}$ & $3.081 \times 10^{-5}$ \\
\hline 35 & & 0.9997 & 0.9998 & $5.092 \times 10^{-5}$ & $2.594 \times 10^{-5}$ \\
\hline 36 & & 0.9986 & 0.9993 & $4.714 \times 10^{-5}$ & $2.338 \times 10^{-5}$ \\
\hline
\end{tabular}




\section{HIERARCHICAL BAYES ESTIMATION OF RELIABILITY INDEXES}

Table 4. Estimates of MTTF and their estimated risks

\begin{tabular}{|c|c|c|c|c|c|}
\hline CS & MTTF & MTTF $_{B}$ & MTTF $_{\text {нв }}$ & $\operatorname{ER}\left(M T T F_{B}\right)$ & $\operatorname{ER}\left(M T T F_{H B}\right)$ \\
\hline 1 & 1.9991 & 2.1663 & 2.1792 & 0.4857 & 0.5213 \\
\hline 2 & & 2.1901 & 2.2039 & 0.5051 & 0.5417 \\
\hline 3 & & 1.9329 & 1.9369 & 0.3798 & 0.4136 \\
\hline 4 & & 2.1270 & 2.1386 & 0.4620 & 0.4965 \\
\hline 5 & & 2.1576 & 2.1702 & 0.4834 & 0.5190 \\
\hline 6 & & 1.8599 & 1.8602 & 0.3788 & 0.4116 \\
\hline 7 & & 2.0519 & 2.0613 & 0.3475 & 0.3687 \\
\hline 8 & & 2.1164 & 2.1276 & 0.3809 & 0.4039 \\
\hline 9 & & 1.8815 & 1.8855 & 0.3062 & 0.3255 \\
\hline 10 & & 1.9954 & 2.0032 & 0.3271 & 0.3473 \\
\hline 11 & & 2.0677 & 2.0776 & 0.3552 & 0.3769 \\
\hline 12 & & 1.7626 & 1.7630 & 0.3027 & 0.3239 \\
\hline 13 & 4.9979 & 5.5000 & 5.5351 & 3.2263 & 3.4580 \\
\hline 14 & & 5.5787 & 5.6162 & 3.4049 & 3.6458 \\
\hline 15 & & 4.8297 & 4.8395 & 2.3392 & 2.5313 \\
\hline 16 & & 5.4589 & 5.4926 & 3.1398 & 3.3672 \\
\hline 17 & & 5.5472 & 5.5838 & 3.3299 & 3.5669 \\
\hline 18 & & 4.6484 & 4.6492 & 2.3318 & 2.5252 \\
\hline 19 & & 5.2490 & 5.2760 & 2.3295 & 2.4697 \\
\hline 20 & & 5.4308 & 5.4626 & 2.6177 & 2.7724 \\
\hline 21 & & 4.7025 & 4.7124 & 1.9184 & 2.2388 \\
\hline 22 & & 5.2357 & 5.2623 & 2.3140 & 2.4531 \\
\hline 23 & & 5.3902 & 5.4209 & 2.5499 & 2.7009 \\
\hline 24 & & 4.4066 & 4.4072 & 1.8230 & 2.1551 \\
\hline 25 & 9.9958 & 11.1494 & 11.2244 & 13.5400 & 14.4996 \\
\hline 26 & & 11.1964 & 11.2729 & 13.7659 & 14.7371 \\
\hline 27 & & 9.6457 & 9.6649 & 9.2994 & 10.1653 \\
\hline 28 & & 11.0364 & 11.1081 & 12.9450 & 13.8712 \\
\hline 29 & & 11.1528 & 11.2282 & 13.4762 & 14.4297 \\
\hline 30 & & 9.2739 & 9.2750 & 9.2443 & 10.1482 \\
\hline 31 & & 10.8628 & 10.9265 & 10.5038 & 11.1230 \\
\hline 32 & & 10.9300 & 10.9954 & 10.7570 & 11.3888 \\
\hline 33 & & 9.4016 & 9.4214 & 7.6860 & 8.1665 \\
\hline 34 & & 10.7068 & 10.7662 & 10.0085 & 10.6043 \\
\hline 35 & & 10.8999 & 10.9644 & 10.6881 & 11.3177 \\
\hline 36 & & 8.8144 & 8.8156 & 7.0966 & 7.6261 \\
\hline
\end{tabular}




\section{BAROT \& PATEL}

Table 5. The effect of $k$ on the estimates of reliability indexes

\begin{tabular}{|c|c|c|c|c|c|c|c|}
\hline CS & $k$ & $\hat{\lambda}_{B}$ & $\hat{\lambda}_{H B}$ & $\hat{\mathbf{R}}(\boldsymbol{t})_{B}$ & $\hat{\mathbf{R}}(\boldsymbol{t})_{\text {нв }}$ & MTTF $_{B}$ & MTTF $_{\text {Hв }}$ \\
\hline \multirow[t]{3}{*}{1} & 4 & 2.2951 & 2.3031 & 0.6261 & 0.6282 & 2.7078 & 2.7240 \\
\hline & 8 & & & 0.1543 & 0.1587 & 1.3539 & 1.3620 \\
\hline & 12 & & & 0.0371 & 0.0388 & 0.9026 & 0.9080 \\
\hline \multirow[t]{3}{*}{4} & 4 & 2.3396 & 2.3504 & 0.6118 & 0.6136 & 2.6588 & 2.6732 \\
\hline & 8 & & & 0.1464 & 0.1506 & 1.3294 & 1.3366 \\
\hline & 12 & & & 0.0346 & 0.0362 & 0.8863 & 0.8911 \\
\hline \multirow[t]{3}{*}{7} & 4 & 2.3322 & 2.3365 & 0.6095 & 0.6115 & 2.5648 & 2.3365 \\
\hline & 8 & & & 0.1210 & 0.1244 & 1.2824 & 1.2883 \\
\hline & 12 & & & 0.0225 & 0.0236 & 0.8549 & 0.8589 \\
\hline \multirow[t]{3}{*}{10} & 4 & 2.3984 & 2.4059 & 0.5866 & 0.5881 & 2.4943 & 2.5040 \\
\hline & 8 & & & 0.1099 & 0.1129 & 1.2471 & 1.2520 \\
\hline & 12 & & & 0.0198 & 0.0206 & 0.8314 & 0.8346 \\
\hline \multirow[t]{3}{*}{13} & 4 & 2.2612 & 2.2675 & 0.9896 & 0.9873 & 6.8750 & 6.9188 \\
\hline & 8 & & & 0.8067 & 0.8056 & 3.4375 & 3.4594 \\
\hline & 12 & & & 0.5095 & 0.5144 & 2.2917 & 2.3063 \\
\hline \multirow[t]{3}{*}{16} & 4 & 2.2779 & 2.2850 & 0.9893 & 0.9869 & 6.8236 & 6.8658 \\
\hline & 8 & & & 0.8022 & 0.8010 & 3.4118 & 3.4329 \\
\hline & 12 & & & 0.5033 & 0.5080 & 2.2745 & 2.2886 \\
\hline \multirow[t]{3}{*}{19} & 4 & 2.2790 & 2.2808 & 0.9930 & 0.9916 & 6.5613 & 6.5950 \\
\hline & 8 & & & 0.8123 & 0.8115 & 3.2806 & 3.2975 \\
\hline & 12 & & & 0.4877 & 0.4923 & 2.1871 & 2.1983 \\
\hline \multirow[t]{3}{*}{22} & 4 & 2.2845 & 2.2865 & 0.9929 & 0.9915 & 6.5446 & 6.5779 \\
\hline & 8 & & & 0.8108 & 0.8100 & 3.2723 & 3.2889 \\
\hline & 12 & & & 0.4853 & 0.4898 & 2.1815 & 2.1926 \\
\hline \multirow[t]{3}{*}{25} & 4 & 2.2310 & 2.2358 & 0.9999 & 0.9998 & 13.9367 & 14.0305 \\
\hline & 8 & & & 0.9919 & 0.9897 & 6.9684 & 7.0152 \\
\hline & 12 & & & 0.9382 & 0.9348 & 4.6456 & 4.6768 \\
\hline \multirow[t]{3}{*}{28} & 4 & 2.2511 & 2.2564 & 0.9999 & 0.9998 & 13.7955 & 13.8852 \\
\hline & 8 & & & 0.9917 & 0.9896 & 6.8977 & 6.9426 \\
\hline & 12 & & & 0.9362 & 0.9327 & 4.5985 & 4.6284 \\
\hline \multirow[t]{3}{*}{31} & 4 & 2.2031 & 2.2020 & 0.9999 & 0.9999 & 13.5785 & 13.6581 \\
\hline & 8 & & & 0.9955 & 0.9945 & 6.7892 & 6.8290 \\
\hline & 12 & & & 0.9527 & 0.9505 & 4.5261 & 4.5527 \\
\hline \multirow[t]{3}{*}{34} & 4 & 2.2362 & 2.2365 & 0.9999 & 0.9999 & 13.3835 & 13.4578 \\
\hline & 8 & & & 0.9949 & 0.9937 & 6.6917 & 6.7289 \\
\hline & 12 & & & 0.9489 & 0.9465 & 4.4611 & 4.4859 \\
\hline
\end{tabular}




\section{HIERARCHICAL BAYES ESTIMATION OF RELIABILITY INDEXES}

Table 6. The effect of $k$ on the estimated risks of estimates of reliability indexes

\begin{tabular}{|c|c|c|c|c|c|c|c|}
\hline CS & $k$ & $\operatorname{ER}\left(\hat{\lambda}_{B}\right)$ & $\operatorname{ER}\left(\hat{\lambda}_{H B}\right)$ & $\operatorname{ER}\left(\hat{\mathbf{R}}(t)_{B}\right)$ & $\operatorname{ER}\left(\hat{\mathbf{R}}(t)_{H B}\right)$ & $\operatorname{ER}\left(M T T F_{B}\right)$ & $\operatorname{ER}\left(M T T F_{\text {нв }}\right)$ \\
\hline \multirow[t]{3}{*}{1} & 4 & 0.6755 & 0.7867 & 0.0927 & 0.0950 & 0.7590 & 0.8146 \\
\hline & 8 & & & 0.0428 & 0.0454 & 0.1897 & 0.2036 \\
\hline & 12 & & & 0.0043 & 0.0047 & 0.0843 & 0.0905 \\
\hline \multirow[t]{3}{*}{4} & 4 & 0.7288 & 0.8545 & 0.0993 & 0.1018 & 0.7218 & 0.7758 \\
\hline & 8 & & & 0.0394 & 0.0418 & 0.1804 & 0.1939 \\
\hline & 12 & & & 0.0039 & 0.0042 & 0.0802 & 0.0862 \\
\hline \multirow[t]{3}{*}{7} & 4 & 0.6178 & 0.6879 & 0.0985 & 0.1002 & 0.5429 & 0.5761 \\
\hline & 8 & & & 0.0284 & 0.0300 & 0.1357 & 0.1440 \\
\hline & 12 & & & 0.0019 & 0.0021 & 0.0603 & 0.0640 \\
\hline \multirow[t]{3}{*}{10} & 4 & 0.6934 & 0.7772 & 0.1092 & 0.1112 & 0.5111 & 0.5427 \\
\hline & 8 & & & 0.0242 & 0.0256 & 0.1278 & 0.1357 \\
\hline & 12 & & & 0.0015 & 0.0017 & 0.0568 & 0.0603 \\
\hline \multirow[t]{3}{*}{13} & 4 & 0.6451 & 0.7537 & 0.0015 & 0.0026 & 5.0411 & 5.4031 \\
\hline & 8 & & & 0.0790 & 0.0829 & 1.2603 & 1.3508 \\
\hline & 12 & & & 0.0871 & 0.0891 & 0.5601 & 0.6003 \\
\hline \multirow[t]{3}{*}{16} & 4 & 0.6593 & 0.7669 & 0.0015 & 0.0027 & 4.9060 & 5.2612 \\
\hline & 8 & & & 0.0815 & 0.0856 & 1.2265 & 1.3153 \\
\hline & 12 & & & 0.0882 & 0.0902 & 0.5451 & 0.5846 \\
\hline \multirow[t]{3}{*}{19} & 4 & 0.5607 & 0.6197 & 0.0009 & 0.0014 & 3.6399 & 3.8589 \\
\hline & 8 & & & 0.0752 & 0.0782 & 0.9100 & 0.9647 \\
\hline & 12 & & & 0.0804 & 0.0818 & 0.4044 & 0.4288 \\
\hline \multirow[t]{3}{*}{22} & 4 & 0.5640 & 0.6223 & 0.0009 & 0.0014 & 3.6156 & 3.8330 \\
\hline & 8 & & & 0.0759 & 0.0790 & 0.9039 & 0.9582 \\
\hline & 12 & & & 0.0808 & 0.0821 & 0.4017 & 0.4259 \\
\hline \multirow[t]{3}{*}{25} & 4 & 0.6177 & 0.7183 & $2.071 \times 10^{-6}$ & $3.023 \times 10^{-5}$ & 21.1563 & 22.6557 \\
\hline & 8 & & & 0.0012 & 0.0023 & 5.2891 & 5.6639 \\
\hline & 12 & & & 0.0176 & 0.0210 & 2.3507 & 2.5173 \\
\hline \multirow[t]{3}{*}{28} & 4 & 0.6259 & 0.7249 & $1.565 \times 10^{-6}$ & $2.568 \times 10^{-5}$ & 20.2266 & 21.6737 \\
\hline & 8 & & & 0.0012 & 0.0022 & 5.0566 & 5.4184 \\
\hline & 12 & & & 0.0180 & 0.0214 & 2.2474 & 2.4082 \\
\hline \multirow[t]{3}{*}{31} & 4 & 0.4944 & 0.5427 & $4.014 \times 10^{-7}$ & $5.444 \times 10^{-6}$ & 16.4122 & 17.3797 \\
\hline & 8 & & & 0.0006 & 0.0010 & 4.1030 & 4.3449 \\
\hline & 12 & & & 0.0121 & 0.0141 & 1.8236 & 1.9311 \\
\hline \multirow[t]{3}{*}{34} & 4 & 0.5266 & 0.5806 & $3.553 \times 10^{-7}$ & $4.667 \times 10^{-6}$ & 15.6383 & 16.5692 \\
\hline & 8 & & & 0.0007 & 0.0011 & 3.9096 & 4.1423 \\
\hline & 12 & & & 0.0136 & 0.0159 & 1.7376 & 1.8410 \\
\hline
\end{tabular}

\section{Numerical Examples}

Two numerical examples are presented to illustrate how the data support the developed model and how to employ the proposed method for estimation of reliability indexes of the series system. Examples 1 and 2 consider the artificial 


\section{BAROT \& PATEL}

general progressive Type II censored samples generated from the real data set provided by Nelson (1982) and the computer simulation, respectively.

\section{Example 1. Real Life Data}

As a numerical illustration, a system comprising a series of 2 working units and 18 cold standby units was considered. This series system is equivalent to a cold standby series system of 19 identical and independent units. The lifetimes of such 19 units were observed until failure during the life test experiment in which specimens of a type of electrical insulating fluid were subject to a constant voltage stress ( $34 \mathrm{KV} /$ minutes). The 19 failure times were obtained as follows:

$$
\begin{array}{cccccccccc}
0.19 & 0.78 & 0.96 & 1.31 & 2.78 & 3.16 & 4.15 & 4.67 & 4.85 & 6.50 \\
7.35 & 8.01 & 8.27 & 12.06 & 31.75 & 32.52 & 33.94 & 36.71 & 72.89
\end{array}
$$

Asgharzadeh and Valiollahi (2009) checked the validity of an exponential model with mean $=14.2857$ and indicated that the exponential model is adequate for this data set. To generate an artificial general progressive Type II censored sample from the given real data set, it is assumed that the lifetimes of the first two failures are lost without observation, and then lifetimes were observed until the eighth failure. At each failure from $3^{\text {rd }}$ failure to $8^{\text {th }}$ failure, units were randomly withdrawn according to the general progressive Type II censoring scheme $\mathbf{r}=\left(r_{3}, r_{4}, \ldots, r_{8}\right)$ $=(2,0,1,2,1,5)$. The life test was terminated at the eighth failure, and the vector of observed lifetimes was found to be $\mathbf{x}=\left(x_{(3)}, x_{(4)}, \ldots, x_{(8)}\right)$ $=(0.96,1.31,2.78,4.85,6.50,8.01)$.

Table 7. Estimates of reliability indexes and their $(1-\alpha) \%$ HPD-intervals for Example 1

\begin{tabular}{rrrrr} 
& Parameter & Estimate & 95\% HPD-interval & 99\% HPD-interval \\
\hline Bayes Estimation & & 0.0519 & $(0.0209,0.0866)$ & $(0.0107,0.1239)$ \\
& $\mathrm{R}(\mathrm{t})$ & 0.9415 & $(0.6257,0.9999)$ & $(0.0992,0.9999)$ \\
& MTTF & 205.5657 & $(109.7102,454.5454)$ & $(76.6798,887.8505)$ \\
\hline Hierarchical & & & & \\
Bayes Estimation & $\mathrm{R}(\mathrm{t})$ & 0.0519 & $(0.0203,0.0880)$ & $(0.0120,0.1172)$ \\
& MTTF & 205.7603 & $(107.9023,467.9803)$ & $(81.0286,791.6666)$ \\
\hline
\end{tabular}




\section{HIERARCHICAL BAYES ESTIMATION OF RELIABILITY INDEXES}

Table 8. Predictive estimates of the remaining lifetimes and their $(1-\alpha) \%$ HPDprediction intervals for Example 1

\begin{tabular}{rrrrr} 
& $\boldsymbol{I}$ & $\boldsymbol{X}(\boldsymbol{(})$ & 95\% HPD-interval & 99\% HPD-interval \\
\hline Bayes Estimation & 9 & 10.1738 & $(8.0262,15.3444)$ & $(8.0206,22.2503)$ \\
& 10 & 12.8786 & $(8.0218,20.7168)$ & $(8.0202,27.9367)$ \\
& 11 & 16.4851 & $(8.0418,28.2399)$ & $(8.0251,38.7317)$ \\
& 12 & 21.8947 & $(8.1446,39.6961)$ & $(8.0996,55.4946)$ \\
& 13 & 32.7139 & $(8.0212,46.9190)$ & $(8.0201,61.6929)$ \\
\hline Hierarchical Bayes & 9 & 10.1758 & $(8.0470,16.1734)$ & $(8.0244,24.3241)$ \\
Estimation & 10 & 12.8833 & $(8.0267,20.7303)$ & $(8.0377,27.9939)$ \\
& 11 & 16.4931 & $(8.0355,28.2591)$ & $(8.0298,38.7608)$ \\
& 12 & 21.9078 & $(8.0907,39.7260)$ & $(8.0971,55.5395)$ \\
& 13 & 32.7373 & $(8.0261,46.9559)$ & $(8.0241,61.7342)$ \\
\hline
\end{tabular}

The Bayes and hierarchical Bayes estimates of failure rate $\lambda$, reliability $\mathrm{R}(t)$, and MTTF and the corresponding HPD-intervals at $t=100$ have been computed, and are reported in Table 7. The 95\% and 99\% Bayes and hierarchical Bayes predictive estimates and the corresponding HPD-prediction intervals for the each of the remaining $l$ lifetimes $(9 \leq l \leq)$ have also been computed, and are reported in Table 8.

\section{Example 2. Simulated Data}

As a numerical illustration, a system initiated with the series of 5 working units being in an operational state is placed on a life test along with the other 19 standby units connected in a series. This series system is equivalent to a cold standby series system of 20 identical and independent units. Under a general progressive Type II censoring scheme, the lifetimes of the first two failures are not observed and then the lifetimes are completely observed until the eighth failure. Using the algorithm presented in the previous section, the general progressive Type II censored sample $\mathbf{x}=(0.01250,0.01531,0.02063,0.02679,0.03062,0.05251)$ has been generated with the censoring scheme $\mathbf{r}=(1,0,2,1,2,6)$. For this sample, Bayes and hierarchical Bayes estimates of failure rate $\lambda$, reliability $\mathrm{R}(t)$, and $M T T F$, and the corresponding HPD intervals at $t=2$, have been computed and are reported in Table 9. Moreover, the $95 \%$ and $99 \%$ Bayes and hierarchical Bayes predictive estimates and the corresponding HPD-prediction intervals for each of the remaining $l$ lifetimes $(9 \leq l \leq)$ have also been computed, and are reported in Table 10 . 


\section{BAROT \& PATEL}

Table 9. Estimates of reliability indexes and their $(1-\alpha) \%$ HPD-intervals for Example 2

\begin{tabular}{rrrrr} 
& Parameter & Estimate & 95\% HPD-interval & 99\% HPD-interval \\
\hline Bayes Estimation & $\lambda$ & 2.4747 & $(0.9950,4.1210)$ & $(0.7440,4.8827)$ \\
& $\mathrm{R}(t)$ & 0.3201 & $\left(9.053 \times 10^{-5}, 0.9967\right)$ & $\left(9.9 \times 10^{-7}, 0.9999\right)$ \\
& $M T T F$ & 1.8184 & $(0.9706,4.0201)$ & $(0.8192,5.3763)$ \\
\hline Hierarchical Bayes & & & & \\
Estimation & $\lambda$ & 2.4650 & $(0.9910,4.1050)$ & $(0.74800,4.8390)$ \\
& $\mathrm{R}(t)$ & 0.3235 & $\left(9.895 \times 10^{-5}, 0.9968\right)$ & $\left(1.31 \times 10^{-6}, 0.9999\right)$ \\
& $M T T F$ & 1.8256 & $(0.9744,4.0363)$ & $(0.82661,5.3476)$ \\
\hline
\end{tabular}

Table 10. Predictive estimates of the remaining lifetimes and their $(1-\alpha) \%$ HPDprediction intervals for Example 2

\begin{tabular}{rrrrr} 
& $\boldsymbol{I}$ & $\boldsymbol{X}(\boldsymbol{( )}$ & 95\% HPD-interval & 99\% HPD-interval \\
\hline Bayes Estimation & 9 & 0.0676 & $(0.0527,0.1079)$ & $(0.0526,0.1661)$ \\
& 10 & 0.0858 & $(0.0526,0.1394)$ & $(0.0526,0.1888)$ \\
& 11 & 0.1086 & $(0.0530,0.1859)$ & $(0.0526,0.2546)$ \\
& 12 & 0.1389 & $(0.0526,0.2477)$ & $(0.0526,0.3431)$ \\
& 13 & 0.1843 & $(0.0562,0.3429)$ & $(0.0526,0.4821)$ \\
\hline Hierarchical Bayes & 9 & & & \\
Estimation & 10 & 0.0677 & $(0.0529,0.1201)$ & $(0.0526,0.1662)$ \\
& 11 & 0.0860 & $(0.0527,0.1398)$ & $(0.0526,0.1893)$ \\
& 12 & 0.1398 & $(0.0526,0.1864)$ & $(0.0526,0.2554)$ \\
& 13 & 0.1848 & $(0.0531,0.2485)$ & $(0.0526,0.3442)$ \\
& & $(0.0526,0.3440)$ & $(0.0526,0.4838)$ \\
\hline
\end{tabular}

From the results presented in Tables 7-10, it is observed that the hierarchical Bayes estimates and predictors are very close to the Bayes estimates and predictors for both the considered real and simulated data. Furthermore, the Bayes and hierarchical Bayes predictive estimates and the length of the HPD-prediction interval increases as $l$ increases. This implies that the prediction is less precise as a large $l$ is considered.

\section{Conclusion}

This purpose of this study was to study hierarchical Bayes estimation and prediction of reliability indexes and remaining lifetimes of a cold standby series system consisting a series of $k$ working units and $(n-1)$ cold standby units under general progressive Type II censoring scheme. The Bayes and hierarchical Bayes estimates as well as an HPD interval for reliability indexes of the series system are derived. In addition, we have derived the Bayes and hierarchical Bayes predictive estimates, 


\section{HIERARCHICAL BAYES ESTIMATION OF RELIABILITY INDEXES}

and HPD-prediction interval for the remaining lifetimes based on an informative sample. We have presented two numerical examples to illustrate the proposed estimation and prediction methods. The Monte Carlo simulation study is carried out to examine and compare the performance of the Bayes and hierarchical Bayes estimates. The simulation results indicated Bayes estimation should be preferred over the hierarchical Bayes estimation for estimation of reliability indexes of the series system. Furthermore, the number of components in the working condition should be less and the number of components in the cold standby mode should be large to run the series system for a long period.

\section{References}

Ali Mousa, M. A. M. (2001). Inference and prediction for Pareto progressively censored data. Journal of Statistical Computation and Simulation, 71(2), 163-181. doi: 10.1080/00949650108812140

Ali Mousa, M. A. M., \& Jaheen, Z. F. (2002). Bayesian prediction for progressively censored data from the Burr model. Statistical Papers, 43, 587-593. doi: 10.1007/s00362-002-0126-7

Asgharzadeh, A., \& Valiollahi, R. (2009). Inference for the proportional hazards family under progressive Type-II censoring. Journal of the Iranian Statistical Society, 8(1-2), 35-53. Retrieved from http://jirss.irstat.ir/article-1-75en.pdf

Bai, C. G., Yu, M. H., \& Hu, S. X. (1998). Multiple Bayes estimates of the reliability performance for cold standby system. Journal of Zhejiang University, 1998(3), 325-328. Available from http://en.cnki.com.cn/Article_en/CJFDTotalZDZC803.011.htm

Balakrishnan, N., \& Sandhu, R. A. (1996). Best linear unbiased and maximum likelihood estimation for exponential distributions under general progressive Type II censored samples. Sankhyā: The Indian Journal of Statistics, Series B, 58(1), 1-9. Available from http://www.jstor.org/stable/25052923

Barot, D. R., \& Patel M. N. (2014). Bayesian estimation of reliability indexes for cold standby system under general progressive Type II censored data. International Journal Quality \& Reliability Management, 31(3), 311-343. doi: 10.1108/IJQRM-08-2012-0114

Cao, J., \& Cheng, K. (1986). Ke kao xing shu xue yin lun [The theory of reliability mathematics]. Beijing: Science Press. 


\section{BAROT \& PATEL}

Chhikara, R. S., \& Guttman, I. (1982). Prediction limits for the inverse Gaussian distribution. Technometrics, 24(4), 319-324. doi:

10.1080/00401706.1982.10487794

Dunsmore, I. R. (1974). The Bayesian predictive distribution in life testing models. Technometrics, 16(3), 455-460. doi: 10.1080/00401706.1974.10489216

Fernández, A. J. (2000). Bayesian inference from type II doubly censored Rayleigh data. Statistics \& Probability Letters, 48(4), 393-399. doi: 10.1016/S0167-7152(00)00021-3

Fernández, A. J. (2004). On estimating exponential parameters with general Type II progressive censoring. Journal of Statistical Planning and Inference, 121(1), 135-147. doi: 10.1016/S0378-3758(03)00083-1

Han, M. (1998). Wu shi xiao shu ju de duo ceng Bayes ke kao xing fen xi [The hierarchical Bayesian reliability analysis of zero-failure data]. Mathematica Applicata, 11(2), 131-134.

Howlader, H. A. (1985). HPD prediction intervals for Rayleigh distribution. IEEE Transactions on Reliability, R-34(2), 121-123. doi:

10.1109/TR.1985.5221968

Kim, C., \& Han, K. (2009). Estimation of the scale parameter of the Rayleigh distribution under general progressive censoring. Journal of the Korean Statistical Society, 38(3), 239-246. doi: 10.1016/j.jkss.2008.10.005

Lehmann, E. L., \& Casella, G. (1998). Theory of point estimation. New York: Springer.

Mei, Q., Liao, J., \& Sun, H. (1992). Kěkào xìng gōngchéng de gāi xìtǒng de $j \bar{l} c h \breve{u}$ [The foundation of reliability engineering for the system]. Beijing: Science Press.

Nelson, W. (1982). Applied life data analysis. New York: Wiley.

Papadopoulos, A. S., Tiwari, R. C., \& Zalkikar, J. N. (1996). Hierarchical Bayes estimation for the exponential-multinomial model in reliability and competing risks. IEEE Transactions on Reliability, 45(3), 477-484. doi: $10.1109 / 24.537019$

Pham, T. G., \& Turkkan, N. (1994). Reliability of a standby system with beta-distributed component lives. IEEE Transactions on Reliability, 43(1), 71-75. doi: 10.1109/24.285114

Raqab, M. Z., \& Madi, M. T. (2002). Bayesian prediction of the total time on test using doubly censored Rayleigh data. Journal of Statistical Computation and Simulation, 72(10), 781-789. doi: 10.1080/00949650214670 


\section{HIERARCHICAL BAYES ESTIMATION OF RELIABILITY INDEXES}

Su, Y., \& Gu, G. (2003). Bayes estimation for reliability indexes of cold

standby system. Journal of North China Electric Power University, 30(2), 96-99. Available from http://en.cnki.com.cn/Article_en/CJFDTOTALHBDL200302022.htm

Willits, C. J. (1997). Series-system reliability-estimation using very small binomial samples. IEEE Transactions on Reliability, 46(2), 296-301. doi: 10.1109/24.589960

Xu, Y., Kang, H., \& Shi, Y. (2002). Bayesian and multiple Bayesian analysis of the reliability performances for series system with cold standby units. Chinese quarterly Journal of Mathematics, 17(2), 26-30. Retrieved from http://166.111.121.20:9080/mathjournal/SXJK200202/sxjk200202005.caj.pdf

Younes, H., Delampady, M., MacGibbon, B., \& Cherkaoui, O. (2007). A hierarchical Bayesian approach to the estimation of monotone hazard rates in the random right censorship model. Journal of Statistical Research, 41(2), 35-62. Retrieved from http://www.isrt.ac.bd/sites/default/files/jsrissues/v41n2/v41n2p35.pdf 